\title{
Intracorporeal versus extracorporeal urinary diversion in robot-assisted radical cystectomy: a systematic review and meta-analysis
}

\author{
Satoshi Katayama ${ }^{1,2}\left(\mathbb{0} \cdot\right.$ Keiichiro Mori ${ }^{1,3} \cdot$ Benjamin Pradere ${ }^{1,4} \cdot$ Hadi Mostafaei ${ }^{1,5} \cdot$ Victor M. Schuettfort $^{1,6}$. \\ Fahad Quhal $^{1,7}$. Reza Sari Motlagh ${ }^{1,8}$. Ekaterina Laukhtina ${ }^{1,9} \cdot$ Marco Moschini $^{10}$. Nico C. Grossmann ${ }^{1,11}$. \\ Yasutomo Nasu ${ }^{2}$. Shahrokh F. Shariat ${ }^{1,9,12,13,14,15,16} \cdot$ Harun Fajkovic ${ }^{1,15}$
}

Received: 26 March 2021 / Accepted: 13 June 2021 / Published online: 19 June 2021

(c) The Author(s) 2021

\begin{abstract}
This systematic review and meta-analysis aimed to assess and compare the perioperative and oncological outcomes of intracorporeal (ICUD) and extracorporeal (ECUD) urinary diversion following robot-assisted radical cystectomy (RARC). A systematic literature search of articles was performed in PubMed ${ }^{\circledR}$, Web of Science ${ }^{\circledR}$, and Scopus ${ }^{\circledR}$ databases according to the Preferred Reporting Items for Systematic Review and Meta-Analysis statement. We included studies that compared patients who underwent RARC with ICUD to those with ECUD. Twelve studies including 3067 patients met the eligibility criteria. There were no significant differences between ICUD and ECUD in overall and major complications, regardless of the period (short-term [ $\leq 30$ days] or mid-term [ $>30$ days]). Subgroup analyses demonstrated that ICUD performed by high-volume centers exhibited a significantly reduced risk of major complications (short-term: OR 0.57, 95\% CI 0.37-0.86, $p=0.008$, mid-term: OR 0.66, 95\% CI 0.46-0.94, $p=0.02$ ). Patients who underwent ICUD had lower estimated blood loss (MD -102.3 ml, 95\% CI - 132.8 to - 71.8, $p<0.00001$ ), less likely to receive blood transfusion rates (OR 0.36, 95\% CI $0.20-0.62, p=0.00003$ ); and these findings were consistent in subgroup analyses by low-volume centers (MD- $121.6 \mathrm{ml}$, $95 \%$ CI -160.9 to $-82.3, p<0.00001$ and OR $0.36,95 \%$ CI $0.20-0.62, p=0.00003$, respectively). ICUD had a higher lymph node yield (MD 3.68, 95\% CI 0.80-6.56, $p=0.01$ ). Patients receiving ICUD provided comparable complications, superior perioperative outcomes, and similar oncological outcomes compared with ECUD. Centralization of patients may contribute to a reduction of postoperative complications, while maintaining the advantages.
\end{abstract}

Keywords Robot-assisted radical cystectomy $\cdot$ Intracorporeal urinary diversion · Extracorporeal urinary diversion · Complication $\cdot$ Hospital volume $\cdot$ Meta-analysis

\section{Introduction}

Radical cystectomy with pelvic lymph node (LN) dissection is the mainstay of treatment for patients with muscle-invasive bladder cancer and very high-risk non-muscle-invasive bladder cancer [1, 2]. Since its advent in 2003, the adoption of robot-assisted radical cystectomy (RARC) has increased steadily worldwide with the promise to lower complications and improve perioperative outcomes, while receiving oncologic efficacy [3-7]. Randomized controlled trials (RCTs)

Harun Fajkovic

harun.fajkovic@meduniwien.ac.at

Extended author information available on the last page of the article revealed that RARC with extracorporeal urinary diversion (ECUD), the initial type of urinary diversion (UD), did not improve complication rates compared to open radical cystectomy (ORC) $[8,9]$. ICUD has been introduced with the aim to fulfill the promise of RARC to reduce complications, including bowel occlusion due to limited manipulation and air exposure, wound-related complications, and anastomotic stricture $[10,11]$. To date, some observational studies suggest an advantage to ICUD over EUCD [12, 13], but no randomized trial has yet compared the differences between these two types of UD. Indeed, due to the heterogeneity in patient population and surgical factors, such a trial will be challenging because of the needed sample size and randomization. Recently, the International Robotic Cystectomy Consortium (IRCC) database, although this cohort consisted 
of 26 institutions, reported that intracorporeal urinary diversion (ICUD) use increased dramatically by an $11 \%$ increase rate per year, from $9 \%$ in 2005 to $97 \%$ in 2016 [14]. However, due to this data from leading experienced institutions, it may not represent real-world data. Since these retrospective studies are subject to inherent recruitment and allocation bias, it might not reflect the true impact of ICUD. One major concern for ICUD has caused by an increased risk of perioperative morbidity with prolonged operative time due to its highly complex procedure. Thereby, whether a wide variety of institutions should willingly incorporate ICUD at the cost of the great disadvantage remains unclear. For an understanding of the current situation in the impact of ICUD, we performed a systematic review and meta-analysis of the literature comparing complications as well as perioperative and oncological outcomes between ICUD and ECUD. Moreover, we evaluated the effect on hospital volume of this complex procedure by performing subgroup analyses. The provided data should help set up a framework for discussion and trial planning.

\section{Patients and methods}

This study is registered with the International Prospective Register of Systematic Reviews (CRD: 42020212880).

\section{Literature search strategy}

The present systematic review and meta-analysis were performed according to PRISMA (the Preferred Reporting Items for Systematic Reviews and Meta-Analyses) statement [15]. A comprehensive literature search using the electronic database (PubMed, Web of Science, and Scopus) was carried out in September 2020 to retrieve published articles comparing complications, perioperative and oncological outcomes of patients who underwent RARC with ICUD to those who underwent RARC with ECUD. The search for eligibility was independently performed by two authors using the following string terms: (urothelial carcinoma OR urothelial cancer OR bladder cancer OR bladder carcinoma) AND (robot-assisted radical cystectomy OR da Vinci radical cystectomy OR robot radical cystectomy) AND (diversion OR ileal conduit OR neobladder) AND (perioperative OR complication OR morbidity OR mortality). The primary outcomes were complications between ICUD and ECUD, graded with the Clavien-Dindo system into overall (grades 1-5) and major (grades 3-5). Concerning the observed period, we divided the complications into short-term ( $\leq 30$ days) and mid-term ( $>30$ days). The secondary outcomes were perioperative outcomes, including operative time, estimated blood loss (EBL), blood transfusion rates, length of stay (LOS), ileus, gastrointestinal (GI)-related complications, and oncological outcomes, including LN yield, number of positive LNs, and soft tissue surgical margin (STSM). After a first screening based on the study title and abstract, the second screening was based on the full-text review and excluded with reasons when deemed inappropriate. Disagreements were resolved via a consensus with coauthors.

\section{Inclusion/exclusion criteria}

The clinical question was established, as stated in the PICO (Population, Intervention, Comparator, Outcome, Study design) approach. Studies were included when bladder cancer patients $(\mathrm{P})$ who had undergone RARC with ICUD (I) as compared with those who had undergone RARC with ECUD (C) in terms of complications, perioperative and oncological outcomes $(\mathrm{O})$ using randomised controlled or observational cohort studies (S). In case of multiple publications on the same cohort, either the high quality or the most recent publication was selected. Reviews, letters, editorials, comments, meeting abstracts, case reports, and articles not published in English were excluded.

\section{Data extraction}

Two authors independently conducted data extraction from the included articles. The extracted data included: first author's name, publication year, period of patient recruitment, recruitment region, study design, number of patients, age, gender, body mass index, neoadjuvant chemotherapy, The American Society of Anesthesiology score, number of surgeons, operative time, EBL, blood transfusion rates, LOS, type of UD, overall and major complications, ileus, GI-related complications, pathologic stage, STSM, LN yield and positive LN. All discrepancies in the data extraction were resolved by consensus with co-investigators.

\section{Statistical analysis}

Forest plots were used as the summary variables for dichotomous and continuous outcomes and to describe the relationships between different UDs and each outcome. Continuous variables are presented as mean \pm standard deviation (SD), and compared with mean differences (MDs). Dichotomous variables are presented as proportions and compared with odds ratios (ORs) and 95\% confidence intervals (CIs). Continuous variables reported as median and interquartile range were altered to mean and SD using the formulas given by Wan et al. [16]. We summarized data using a random-effect model as the studies were heterogeneous. Subsequently, we also performed subgroup analyses according to hospital volume in ICUD. A high-volume center was defined as studies included the number of patients receiving ICUD per hospital per year was $\geq 10$, and a low-volume center was defined as 
the number of patients was $<10$, as recommended by the European Association of Urology Muscle-invasive and Metastatic Bladder Cancer Guideline Panel (EAU MIBC panel) [17]. In the study from IRCC database [18], we estimated the number of patients who underwent ICUD before propensity score matching while referring to the previous study with the same database [14]. Subgroup analyses of complication rates were conducted in studies from high-volume centers as examined the impact of ICUD with reduced the effect of the learning curve. Subgroup analyses of perioperative outcomes were conducted in studies from low-volume centers as examined the adverse effects of ICUD with the learning curve strongly considered. Heterogeneity among the included studies' outcomes in the meta-analysis was evaluated using the $I^{2}$ statistics and the Cochrane $Q$ test. Significant heterogeneity was indicated by an $I^{2}$ test greater than $50 \%$ and $p<0.05$ in the Cochrane $Q$ test. Publication bias was assessed using funnel plots. Statistical analyses were carried out using Review Manager 5.4 (The Nordic Cochrane Centre, Copenhagen, Denmark); the statistical significance level was $p<0.05$.

\section{Risk of bias}

Two authors independently evaluated the risk of bias for all prospective or retrospective cohort studies using the ROBINS-I tool [19]. All discrepancies regarding the risk of bias were resolved by consensus with coauthors.

\section{Results}

\section{Search results}

We identified 298 studies in PubMed, 1659 studies in Web of Science, and 31 studies in Scopus from the initial search; 2 additional studies were added after the latest search. After removing 219 duplicate articles, we screened 1771 titles and abstracts, reviewed 145 full-text articles, which led to the identification of 12 articles comprising 3067 patients that met our inclusion/exclusion criteria [18, 20-30]. Five articles were excluded because of an overlap in the recruitment period at the same institution (Fig. 1). Five and seven studies were divided, respectively, into high-volume center subgroup and low-volume center subgroup. The risk of bias is depicted in Supplementary Table 2.

\section{Included studies}

Overall, 1401 patients (45.7\%) underwent ICUD and 1666 (54.3\%) underwent ECUD. Table 1 demonstrates the basic characteristics of the included studies. Complications, perioperative and oncological outcomes are summarized in
Table 2 and Supplementary Table 1. All studies were nonrandomized controlled studies. In seven studies (58.3\%), RARC plus ICUD were performed by multiple surgeons. Mistretta et al. [30] compared orthotopic ileal neobladder performed intracorporeally vs extracorporeally. Zhang et al. [25] utilized enhance recovery after surgery (ERAS). Hussein et al. [18] used propensity score matching using data from IRCC.

\section{Complications}

\section{Short-term complications}

Nine studies comprising 2459 patients and seven studies comprising 2160 patients were analyzed for overall and major complications, respectively. Forest plots (Fig. 2A, B) showed that there were no significant differences in overall and major complications between ICUD and ECUD (OR $1.08,95 \%$ CI $0.59-1.97, p=0.80$ and OR $1.09,95 \%$ CI $0.58-2.04, p=0.79$, respectively). Between studies, heterogeneities in overall and major complications were significant based on the Cochrane $Q$ test ( $p<0.00001$ and $p=0.01$, respectively) and $I^{2}$ test ( $89 \%$ and $64 \%$, respectively). A subgroup analysis of high-volume centers showed that there was no significant difference in overall (OR 0.75, 95\% CI $0.49-1.14, p=0.18$ ) complications, but ICUD in highvolume centers was significantly associated with a reduced risk of major (OR $0.57,95 \%$ CI $0.37-0.86, p=0.008$ ) complications (Fig. 2C, D). No significant heterogeneities were observed in these subgroup analyses (Cochrane $Q$ test [ $p=0.08$ and $p=0.73]$ and $I^{2}$ [55\% and $\left.0 \%\right]$ in overall and major complications, respectively). The funnel plots for overall and major short-term complications identified four and two studies over the pseudo-95\% CI, respectively (Supplementary Fig. 3A, 3B).

\section{Mid-term complications}

Eight studies comprising 2193 patients and 7 studies comprising 2160 patients were analyzed for overall and major complications, respectively. The forest plots (Fig. 3A, B) showed that there were no significant differences in overall and major complications (OR 0.89, 95\% CI 0.71-1.13, $p=0.34$ and OR $0.94,95 \%$ CI $0.60-1.48, p=0.81$, respectively). Cochrane $Q$ test ( $p=0.82$ and $p=0.22$, respectively) and $I^{2}$ test (0\% and 28\%) showed no significant heterogeneities. A subgroup analysis of high-volume centers showed that there was no significant difference in overall (OR 0.85, 95\% CI 0.64-1.13, $p=0.27$ ) complications, but ICUD in high-volume centers was significantly associated with a reduced risk of major (OR $0.66,95 \%$ CI 0.46-0.94, $p=0.02$ ) complications (Fig. 3C, D). The funnel plot in both 


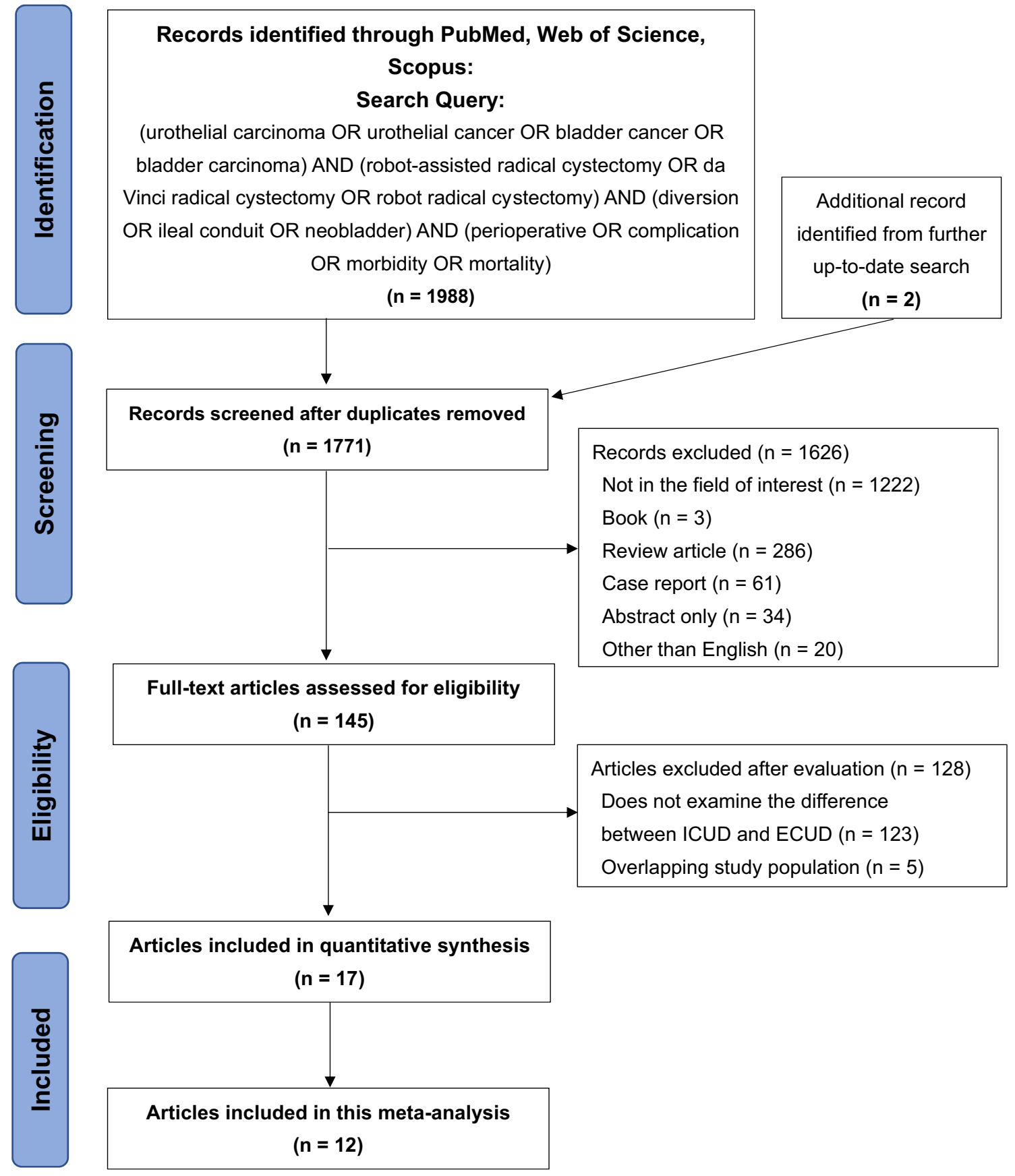

Fig. 1 Flow diagram of the study selection procedure for the systematic review and meta-analysis. ICUD intracorporeal urinary diversion; ECUD extracorporeal urinary diversion

overall and major complications identified no study over the pseudo-95\% CI (Supplementary Fig. 3C, 3D).

\section{Perioperative outcomes}

EBL and blood transfusion rates were significantly lower in patients who underwent an ICUD (MD - $102.3 \mathrm{ml}, 95 \%$ CI -132.8 to $-71.8, p<0.00001$ and OR $0.36,95 \% \mathrm{CI}$
$0.20-0.62, p=0.00003$, respectively) compared to those who underwent an ECUD (Fig. 4A, B). Operative time and LOS were not significantly different between ICUD and ECUD (MD $17.4 \mathrm{~min}, 95 \% \mathrm{CI}-13.2$ to $48.1, p=0.27$ and $\mathrm{MD}-0.87,95 \% \mathrm{CI}-2.12$ to $0.39, p=0.17$, respectively) (Fig. 4C, Supplementary Fig. 1A). Ileus exhibited a marginally trend toward benefit for ICUD but this did not reach statistical significance (OR 0.72, 95\% CI 


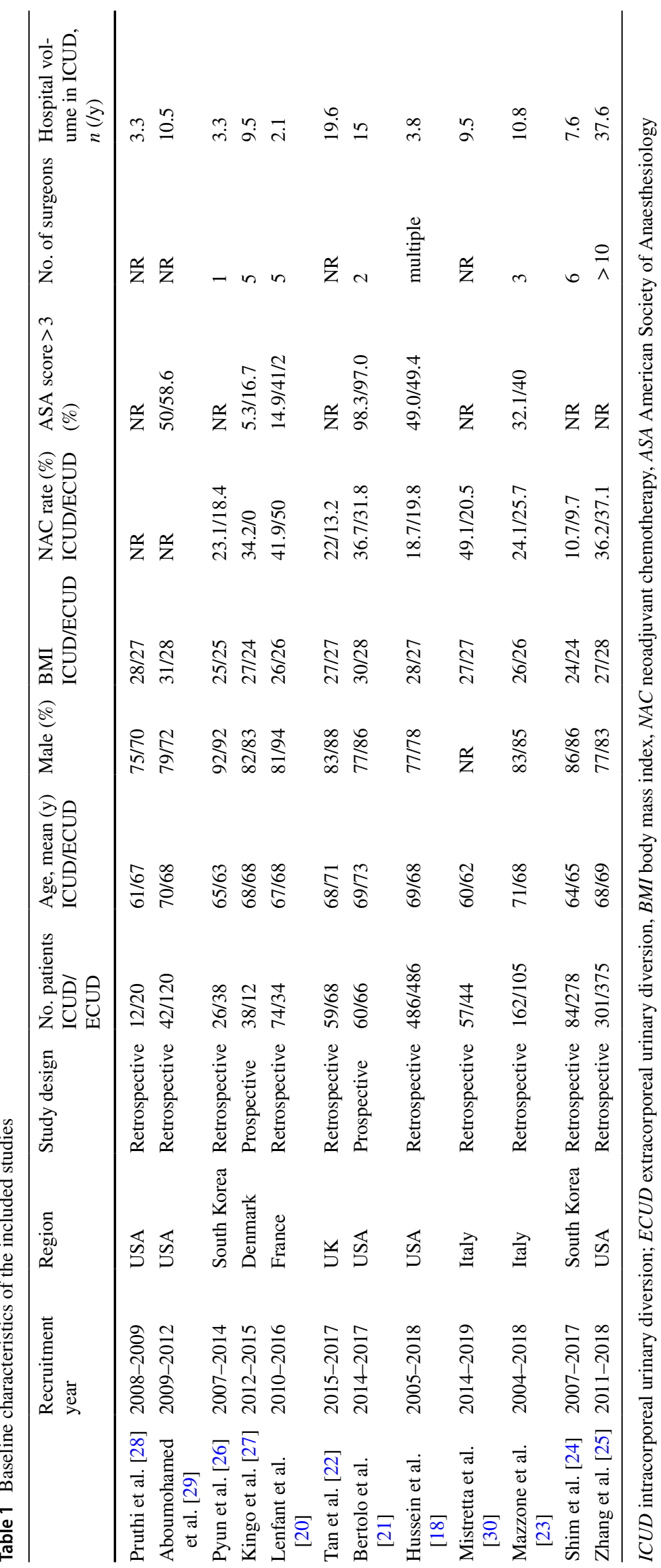


Table 2 Perioperative complications of the included studies

\begin{tabular}{|c|c|c|c|c|c|c|c|}
\hline & $\begin{array}{l}\text { Short-term com- } \\
\text { plication (overall) } \\
(\%)\end{array}$ & $\begin{array}{l}\text { Short-term com- } \\
\text { plication (major) } \\
(\%)\end{array}$ & $\begin{array}{l}\text { Mid-term } \\
\text { complication } \\
\text { (overall) }(\%)\end{array}$ & $\begin{array}{l}\text { Mid-term com- } \\
\text { plication (major) } \\
(\%)\end{array}$ & Ileus (\%) & $\begin{array}{l}\text { Gastrointestinal- } \\
\text { related complica- } \\
\text { tion }(\%)\end{array}$ & $\begin{array}{l}\text { Wound-related } \\
\text { complication } \\
(\%)\end{array}$ \\
\hline Pruthi et al. [28] & $42 / 40$ & NR & $0 / 10$ & NR & NR & NR & NR \\
\hline $\begin{array}{l}\text { Aboumohamed } \\
\text { et al. [29] }\end{array}$ & NR & NR & NR & NR & NR & NR & NR \\
\hline Pyun et al. [26] & NR & NR & NR & NR & NR & $0 / 5$ & $0 / 11$ \\
\hline Kingo et al. [27] & $100 / 100$ & $26 / 0$ & $100 / 100$ & $32 / 8$ & $13 / 8$ & NR & $0 / 0$ \\
\hline Lenfant et al. [20] & $47 / 38$ & $9 / 6$ & $19 / 29$ & $12 / 18$ & NR & NR & NR \\
\hline Tan et al. [22] & $51 / 74$ & $8 / 10$ & $12 / 15$ & $8 / 9$ & NR & NR & NR \\
\hline Bertolo et al. [21] & $22 / 14$ & $2 / 2$ & $7 / 6$ & $2 / 0$ & $5 / 9$ & $10 / 17$ & $0 / 2$ \\
\hline Hussein et al. [18] & $47 / 22$ & $12 / 7$ & $6 / 6$ & $2 / 1$ & NR & $23 / 16$ & $13 / 9$ \\
\hline $\begin{array}{l}\text { Mistretta et al. } \\
{[30]}\end{array}$ & $58 / 59$ & $19 / 20$ & $42 / 36$ & $28 / 25$ & $9 / 7$ & NR & NR \\
\hline $\begin{array}{l}\text { Mazzone et al. } \\
\text { [23] }\end{array}$ & $35 / 43$ & NR & NR & NR & NR & NR & NR \\
\hline Shim et al. [24] & NR & NR & NR & NR & NR & $5 / 13$ & $4 / 3$ \\
\hline Zhang et al. [25] & $38 / 43$ & $10 / 18$ & $44 / 48$ & $17 / 25$ & $21 / 27$ & $23 / 29$ & NR \\
\hline
\end{tabular}

$0.53-1.03, p=0.07$ ) (Supplementary Fig. 1B). There was no difference in GI-related complications between the two UDs (OR 0.75, 95\% CI 0.41-1.39, $p=0.36$ ) (Supplementary Fig. 1C). A subgroup analysis of low-volume centers showed that EBL and blood transfusion rates remained significantly different (MD $-121.6 \mathrm{ml}, 95 \% \mathrm{CI}-160.9$ to $-82.3, p<0.00001$ and OR $0.36,95 \%$ CI $0.20-0.62$, $p=0.00003$, respectively) (Fig. 4D, 4E). Subsequently, operative time and LOS also remained no significantly different in subgroup analyses of low-volume centers (MD $33.3 \mathrm{~min}, 95 \% \mathrm{CI}-16.0$ to $82.7, p=0.19$ and MD $-0.21,95 \% \mathrm{CI}-2.46$ to $2.04, p=0.86$, respectively) (Fig. 4F, Supplementary 2D). The funnel plots identified two studies for EBL, no study for blood transfusion rates, seven studies for operative time, and five studies for LOS over the pseudo-95\% CI (Supplementary Fig. 3E-H, respectively).

\section{Oncological outcomes}

Patients receiving an ICUD had a significantly higher LN yield than those who received an ECUD (MD 3.68, 95\% CI 0.80-6.56, $p=0.01$ ) (Supplementary Fig. 2A). STSM and the positive LN were not significantly different between ICUD and ECUD (OR 1.02, 95\% CI 0.74-1.40, $p=0.92$ and OR $1.12,95 \%$ CI $0.79-1.59, p=0.54$, respectively) (Supplementary Fig. 2B, 2C). The funnel plots identified four studies for LN yield, no study for STSM, and one study for positive LN over the pseudo-95\% CI (Supplementary Fig. 3I, 3J, 3K, respectively).

\section{Discussion}

We performed a systematic review and meta-analysis to evaluate the clinical safety and efficacy of RARC with ICUD compared to ECUD by assessing complications, perioperative and oncological outcomes. Perioperative complications, including overall or major, short-term or mid-term, were comparable between ICUD and ECUD. Subgroup analyses suggested that patients receiving ICUD in high-volume centers had significantly a reduced risk of major complications, but not that of overall complications. Moreover, we found that patients who underwent an ICUD had a significantly lower EBL, lower transfusion rates; these findings remained even in patients from lowvolume centers.

$\mathrm{RC}$ is a technically complex procedure that comprises two major steps: the bladder extirpation phase and the urinary reconstructive phase. With the growing popularity of RARC, the quest for improvement of outcomes has shown some evidence toward better outcomes with ICUD compared to ECUD while maintaining long-term survival outcomes and similar recurrence patterns as well as superior perioperative outcomes and comparable oncological outcomes compared to ORC [31-34]. It is well-known that the urinary reconstructive phase is the major driver of morbidity. By performing the UD intracorporeally, it has been argued that the expected benefits of complete pneumoperitoneum and minimal invasive surgery would lead to tangible improvement for RC patients. Indeed, others and we hypothesized that ICUD might have a positive 
(A)

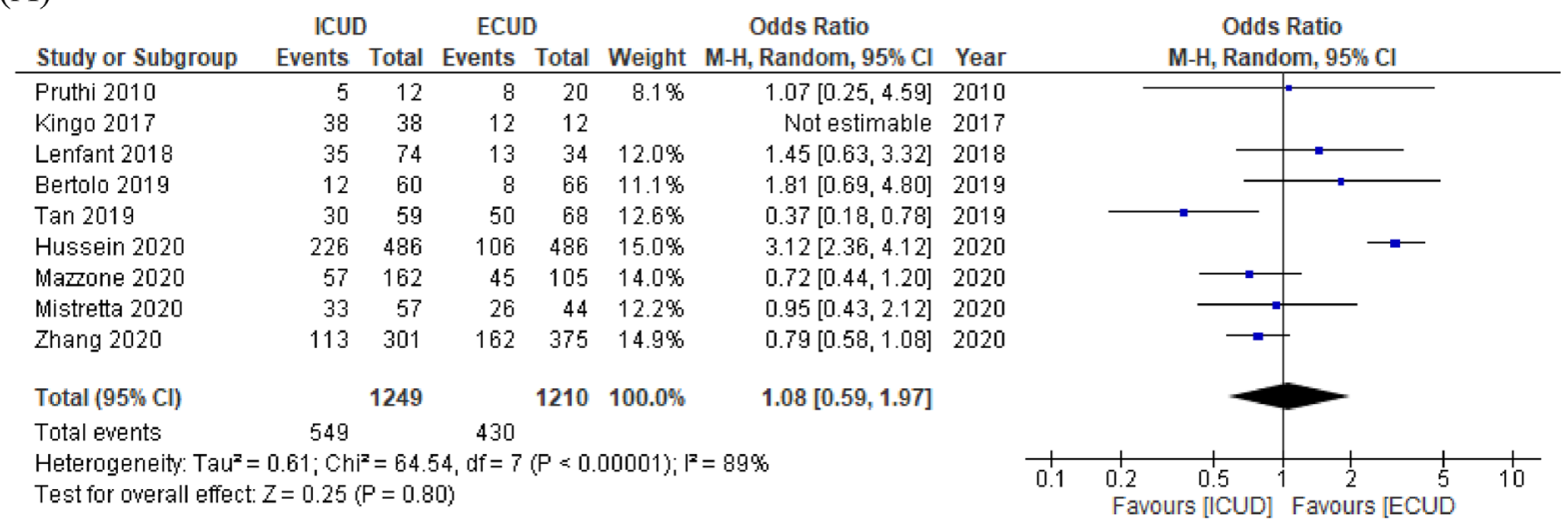

(B)

ICUD ECUD Odds Ratio

- H, Random, $95 \% \mathrm{Cl}$ Year

\begin{tabular}{lrrrrrrr} 
Study or Subgroup & Events & Total & Events & Total & Weight & M-H, Random, 95\% Cl & Year \\
\hline Kingo 2017 & 10 & 38 & 0 & 12 & $4.0 \%$ & $9.21[0.50,169.74]$ & 2017
\end{tabular}

$\begin{array}{llllllll}\text { enfant } 2018 & 7 & 74 & 2 & 34 & 9.8 \% & 1.67[0.33,8.51] 2018\end{array}$

$\begin{array}{rrrrr}7 & 74 & 2 & 34 & 9.8 \% \\ 5 & 59 & 7 & 68 & 14.1 \%\end{array}$

Bertolo 2019

Zhang 2020

Hussein 2020

160

$0.81[0.24,2.69] 2019$

$1.10[0.07,18.01] 2019$

$\begin{array}{lllll}31 & 301 & 67 & 375 & 25.4 \%\end{array}$

$0.53[0.33,0.83] 2020$

$1.79[1.14,2.80] 2020$

$0.93[0.35,2.49] 2020$

Total $(95 \% \mathrm{Cl})$

$56 \quad 486$

$33486 \quad 25.5 \%$

$0.93[0.35,2.49]$

Total events

1075

$1085100.0 \%$

$1.09[0.58,2.04]$

Heterogeneity: Tau $^{2}=0.35 ; \mathrm{Chi}^{2}=16.73, \mathrm{df}=6(\mathrm{P}=0.01) ; \mathrm{I}^{2}=64 \%$

Test for overall effect: $Z=0.27(\mathrm{P}=0.79)$

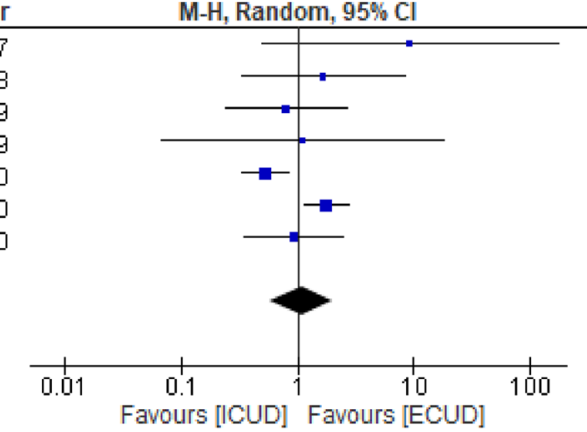

(C)

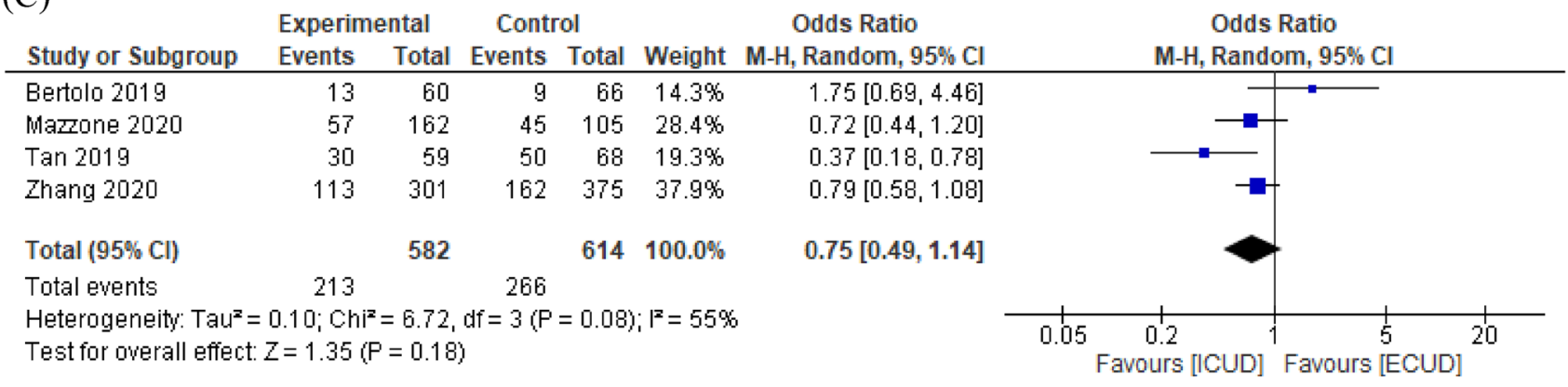

(D)

ICUD ECUD

Odds Ratio

Odds Ratio

Study or Subgroup Events Total Events Total Weight $\mathrm{M}-\mathrm{H}$, Random, $95 \% \mathrm{Cl}$

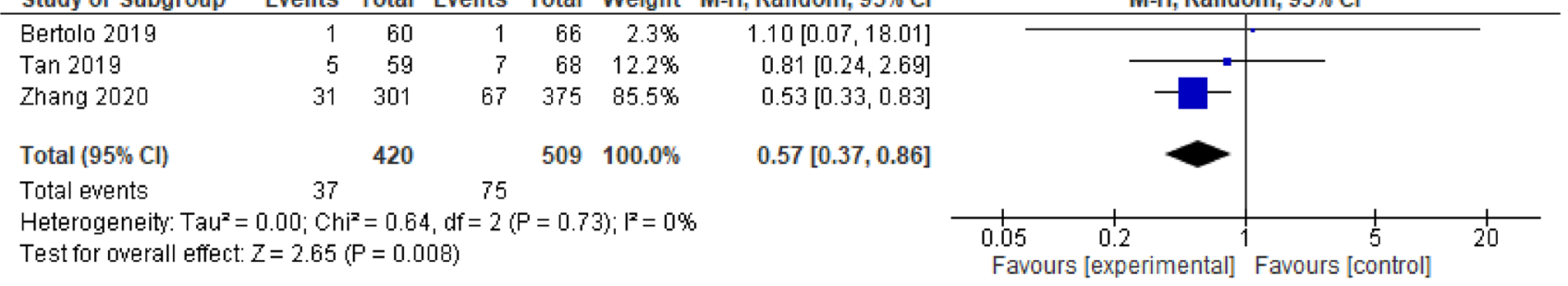

Fig. 2 Forest plots of studies investigating the comparison of $\mathbf{A}$ short-term overall complication, B short-term major complication, and $\mathbf{C}$ subgroup of short-term overall complication, $\mathbf{D}$ subgroup of

short-term major complication classified by hospital volume between RARC with ICUD and RARC with ECUD 


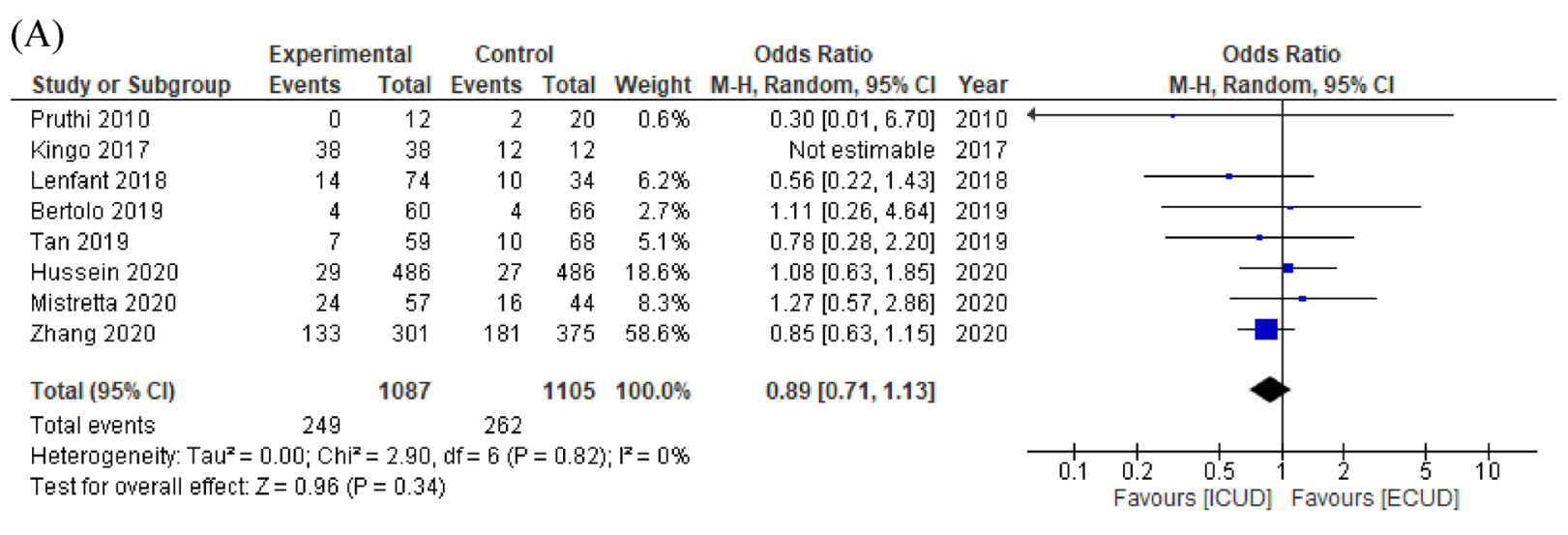

(B)

ICUD ECUD Odds Ratio Study or Subgroup Events Total Events Total Weight M-H, Random, $95 \% \mathrm{Cl}$ Year

\begin{tabular}{lrrrrr}
\hline Kingo 2017 & 12 & 38 & 1 & 12 & $4.0 \%$ \\
Lenfant 2018 & 9 & 74 & 6 & 34 & $12.4 \%$ \\
Tan 2019 & 5 & 59 & 6 & 68 & $10.6 \%$ \\
Bertolo 2019 & 1 & 60 & 0 & 66 & $1.9 \%$ \\
Zhang 2020 & 51 & 301 & 93 & 375 & $39.2 \%$ \\
Hussein 2020 & 10 & 486 & 6 & 486 & $14.4 \%$ \\
Mistretta 2020 & 16 & 57 & 11 & 44 & $17.4 \%$ \\
Total (95\% Cl) & & & & & \\
Total events & 104 & & 1085 & $\mathbf{1 0 0 . 0 \%}$
\end{tabular}

Heterogeneity: $\operatorname{Tau}^{2}=0.10 ; \mathrm{Chi}^{2}=8.30, \mathrm{df}=6(\mathrm{P}=0.22) ; \mathrm{I}^{2}=28 \%$

Test for owerall effect: $Z=0.25(P=0.81)$
$5.08[0.59,43.95] 2017$
$0.65[0.21,1.99] 2018$
$0.96[0.28,3.31] 2019$
$3.35[0.13,83.89] 2019$
$0.62[0.42,0.91] 2020$
$1.68[0.61,4.66] 2020$
$1.17[0.48,2.86] 2020$
$0.94[0.60,1.48]$

(C)

ICUD ECUD

Odds Ratio

Odds Ratio

Study or Subgroup Events Total Events Total Weight M-H, Random, 95\% Cl

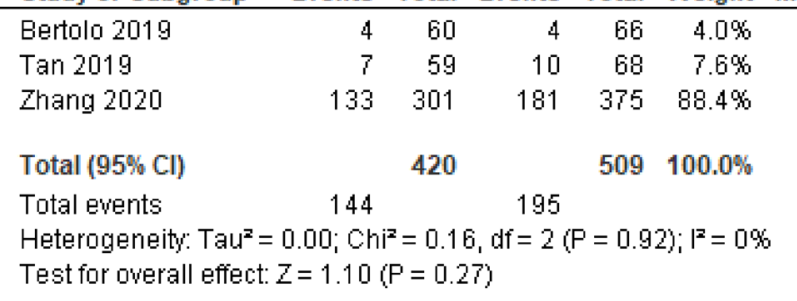

$1.11[0.26,4.64]$

$0.78[0.28,2.20]$

$0.85[0.63,1.15]$

$0.85[0.64,1.13]$

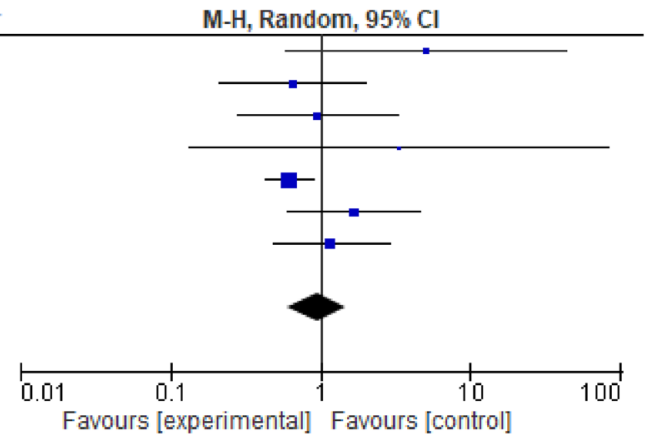

M-H, Random, $95 \% \mathrm{Cl}$

(D)

ICUD ECUD

Odds Ratio

Odds Ratio

Study or Subgroup Events Total Events Total Weight M-H, Random, $95 \% \mathrm{Cl}$

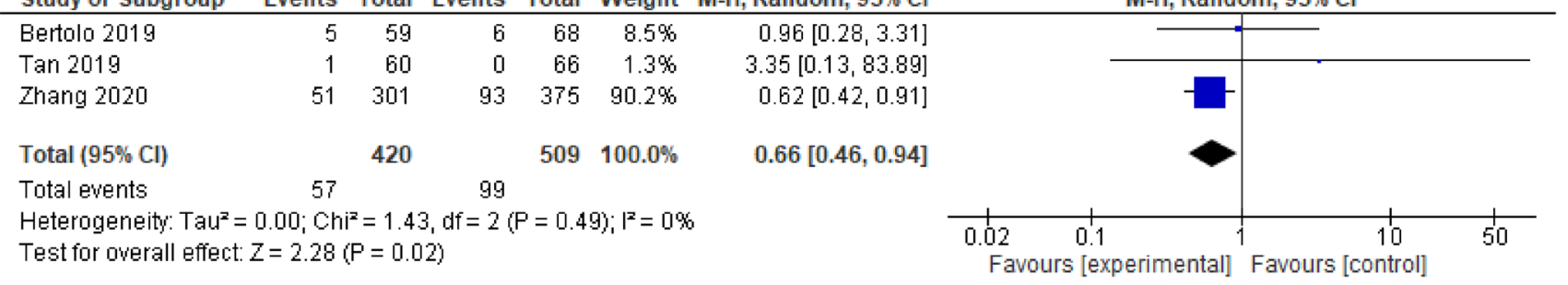

Fig. 3 Forest plots of studies investigating the comparison of $\mathbf{A}$ midterm overall complication, $\mathbf{B}$ mid-term major complication, and $\mathbf{C}$ subgroup of mid-term overall complication, $\mathbf{D}$ subgroup of mid-term major complication classified by hospital volume between RARC with ICUD and RARC with ECUD 


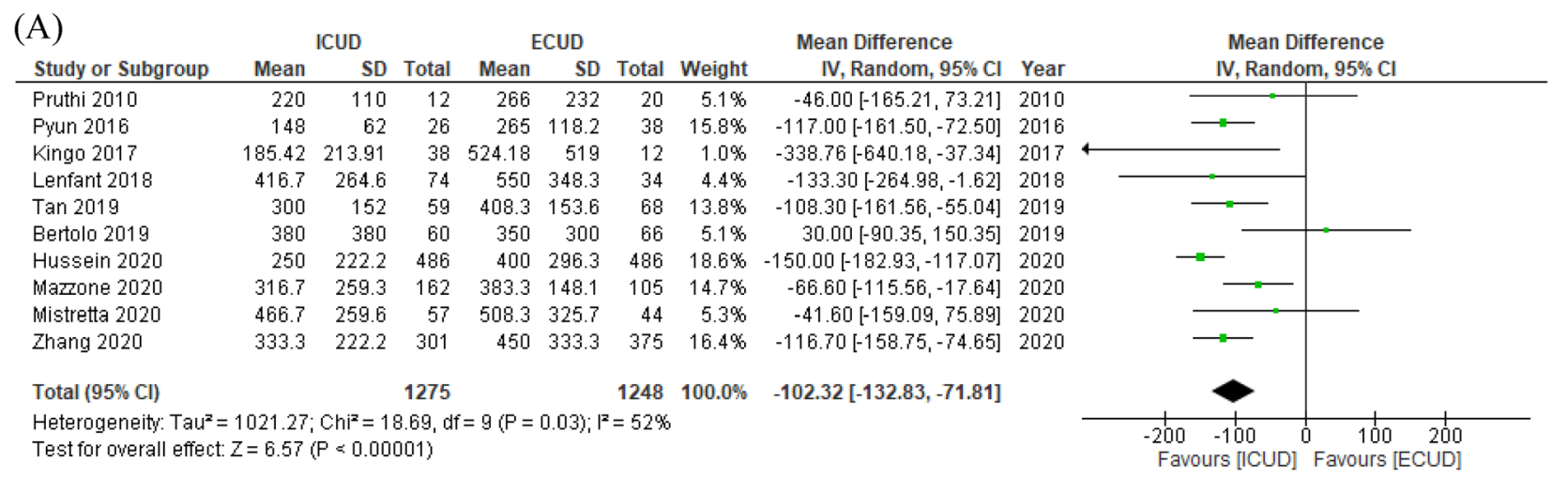

(B)

ICUD ECUD Odds Ratio

Study or Subgroup Events Total Events Total Weight M-H, Random, 95\% Cl Year

\begin{tabular}{lrrrrr} 
Pyun 2016 & 1 & 26 & 11 & 38 & $6.2 \%$ \\
Lenfant 2018 & 4 & 74 & 8 & 34 & $14.3 \%$ \\
Bertolo 2019 & 6 & 60 & 6 & 66 & \\
Mazzone 2020 & 12 & 162 & 11 & 105 & \\
Mistretta 2020 & 1 & 57 & 5 & 44 & $5.9 \%$ \\
Shim 2020 & 13 & 84 & 59 & 278 & $32.0 \%$ \\
Zhang 2020 & 51 & 301 & 91 & 375 & \\
Hussein 2020 & 29 & 486 & 70 & 486 & $41.6 \%$ \\
Total (95\% Cl) & & 727 & & 880 & $100.0 \%$ \\
Total events & 48 & \multicolumn{5}{c}{153} \\
Heterogeneity: Tau $=0.14 ;$ Chi $^{2}=6.54, \mathrm{df}=4(\mathrm{P}=0.16) ; \mathrm{I}^{2}=39 \%$ \\
Test for overall effect: $Z=3.61(\mathrm{P}=0.0003)$
\end{tabular}

$0.10[0.01,0.82] 2016$

$0.19[0.05,0.67] 2018$

Notestimable 2019

Notestimable 2020

$0.14[0.02,1.24] 2020$

$0.68[0.35,1.31] 2020$

Notestimable 2020

$0.38[0.24,0.59] 2020$

$0.36[0.20,0.62]$

\section{$(0.36[0.20,0.62]$}

Test for overall effect: $Z=3.61(\mathrm{P}=0.0003)$
Odds Ratio

M-H, Random, $95 \% \mathrm{Cl}$

(C)

\begin{tabular}{|c|c|c|c|c|c|c|c|c|c|c|c|c|}
\hline \multirow[b]{2}{*}{ Study or Subgroup } & \multicolumn{3}{|c|}{ ICUD } & \multicolumn{3}{|c|}{ ECUD } & \multicolumn{2}{|r|}{ Mean Difference } & \multirow{2}{*}{\multicolumn{4}{|c|}{$\begin{array}{c}\text { Mean Difference } \\
\text { IV, Random, } 95 \% \mathrm{Cl}\end{array}$}} \\
\hline & Mean & SD & Total & Mean & SD & Total & Weight & IV, Random, $95 \% \mathrm{Cl}$ & & & & \\
\hline Pruthi 2010 & 319 & 54 & 12 & 252 & 12 & 20 & $9.0 \%$ & $67.00[36.00,98.00]$ & 2010 & & & \\
\hline Pyun 2016 & 581 & 76.3 & 26 & 468 & 77.7 & 38 & $8.6 \%$ & $113.00[74.65,151.35]$ & 2016 & & & \\
\hline Kingo 2017 & 311.05 & 71.05 & 38 & 332.67 & 79.14 & 12 & $7.8 \%$ & $-21.62[-71.77,28.53]$ & 2017 & & & \\
\hline Lenfant 2018 & 306.7 & 90.7 & 74 & 288.3 & 61.9 & 34 & $9.1 \%$ & $18.40[-10.93,47.73]$ & 2018 & & & \\
\hline Bertolo 2019 & 420 & 60 & 60 & 360 & 60 & 66 & $9.5 \%$ & $60.00[39.02,80.98]$ & 2019 & & & \\
\hline $\operatorname{Tan} 2019$ & 332.7 & 51.7 & 59 & 369.3 & 82.2 & 68 & $9.4 \%$ & $-36.60[-60.17,-13.03]$ & 2019 & & & \\
\hline Hussein 2020 & 356.7 & 92.6 & 486 & 403 & 94.8 & 486 & $9.8 \%$ & $-46.30[-58.08,-34.52]$ & 2020 & & & \\
\hline Mazzone 2020 & 350 & 74.1 & 162 & 383.3 & 148.1 & 105 & $9.0 \%$ & $-33.30[-63.84,-2.76]$ & 2020 & & & \\
\hline Mistretta 2020 & 519.7 & 68 & 57 & 471.7 & 90.4 & 44 & $9.0 \%$ & $48.00[15.98,80.02]$ & 2020 & & & \\
\hline Shim 2020 & 566.7 & 113.7 & 84 & 510.1 & 153.3 & 278 & $9.1 \%$ & $56.60[26.34,86.86]$ & 2020 & & & \\
\hline Zhang 2020 & 400.2 & 81.9 & 301 & 425.3 & 101.5 & 375 & $9.7 \%$ & $-25.10[-38.93,-11.27]$ & 2020 & & & \\
\hline Total $(95 \% \mathrm{Cl})$ & & & 1359 & & & 1526 & $100.0 \%$ & $17.43[-13.24,48.11]$ & & & & \\
\hline $\begin{array}{l}\text { Heterogeneity: Tauz } \\
\text { Test for owerall effect }\end{array}$ & $\begin{array}{l}2467.11 ; \\
Z=1.11\end{array}$ & $\begin{array}{l}\mathrm{Chi}^{2}=1 \\
\mathrm{P}=0.27\end{array}$ & 188.43, & $\mathrm{df}=10$ & $P<0.00$ & $0001) ;$ & $z=95 \%$ & & $\grave{-100}$ & $\begin{array}{c}1 \\
-50 \\
\text { Favours [ICUD] }\end{array}$ & $\begin{array}{cc} & 50 \\
\text { Favours [ECUD] }\end{array}$ & 100 \\
\hline
\end{tabular}

(D)

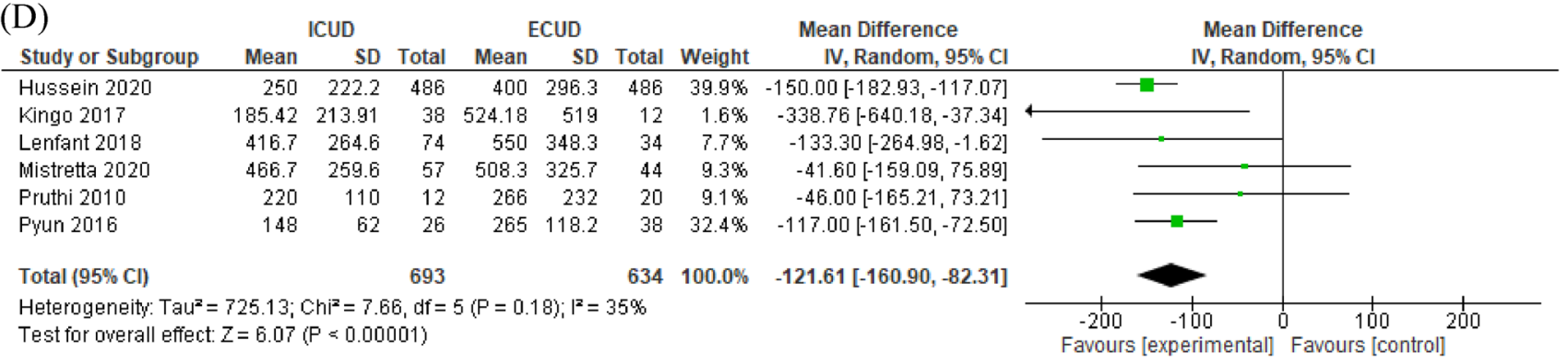

Fig. 4 Forest plots of studies investigating the comparison of A estimate blood loss, B blood transfusion rates, $\mathbf{C}$ operative time, and $\mathbf{D}$ subgroup of estimate blood loss, E subgroup of blood transfusion rates, F subgroup of operative time classified by hospital volume between RARC with ICUD and RARC with ECUD 


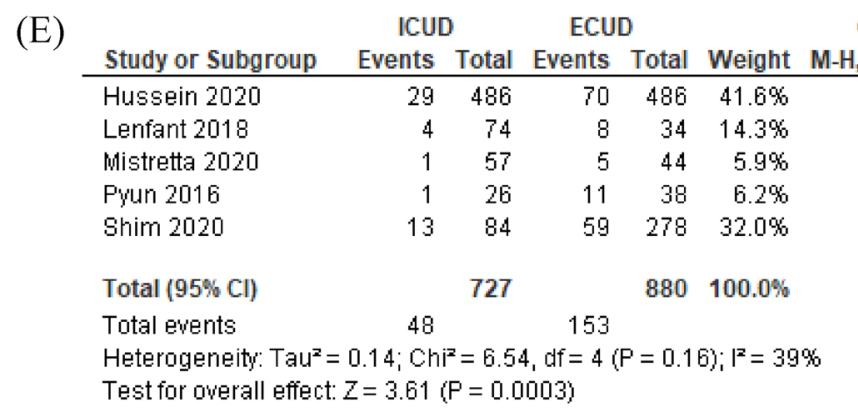

(F)

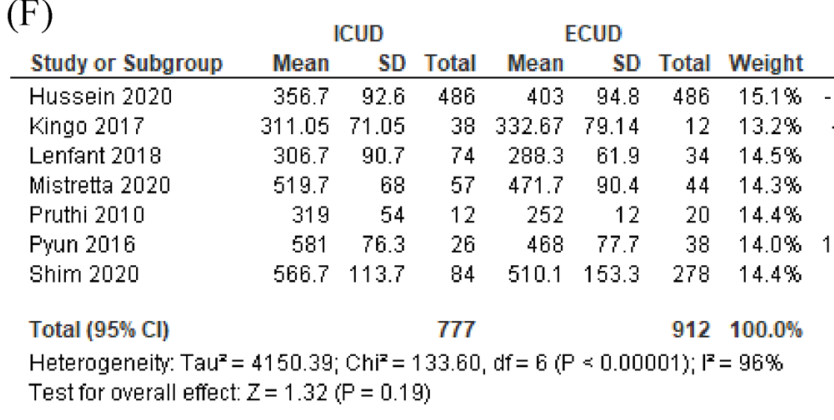

Fig. 4 (continued)

impact in terms of postoperative complications compared to ECUD. We found, however, no difference in overall and major complications between ICUD and ECUD in our meta-analysis. For this reason, it may be conceivable that heterogeneity in the learning curve proficiency in the reconstructive phase affected these analyses. To flatten the learning curve in the extirpation phase, at least 30 cases are needed, which leads to reduced EBL, less STSM, or an adequate number of retrieved $\operatorname{LN}[35,36]$. However, it may be argued that the reconstructive phase needs a much longer learning curve to obtain the best possible complication rates compared to the extirpative phase [36]. Most urologists initially adopt ECUD, as they were familiar with this technique from the open approach. Over the years, the lack of significant benefit offered by ECUD resulted in a push to transition to ICUD. In this regard, almost all studies included in this meta-analysis represented the results comparing the first ICUD cases to the last ECUD cases. Considering that RARC is not a high-volume surgery, unlike robot-assisted radical prostatectomy, it appears to be evident that hospital volume was associated with perioperative complications, mortality, and long-term oncologic outcomes [17, 37]. Thus, EAU MIBC panel recently advocated a threshold number of RCs per hospital and/or surgeon (at least 10, and preferably $>20$ ), being highlighted the potential benefit of centralization of RARC [17]. Despite a lack of high level evidence, further centralization of ICUD may be needed
Odds Ratio Odds Ratio

$0.38[0.24,0.59]$

$0.19[0.05,0.67]$

$0.14[0.02,1.24]$

$0.10[0.01,0.82]$

$0.68[0.35,1.31]$

$0.36[0.20,0.62]$

M-H, Random, $95 \% \mathrm{Cl}$

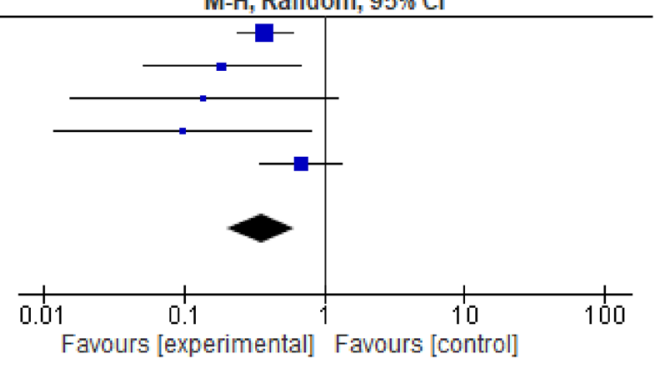

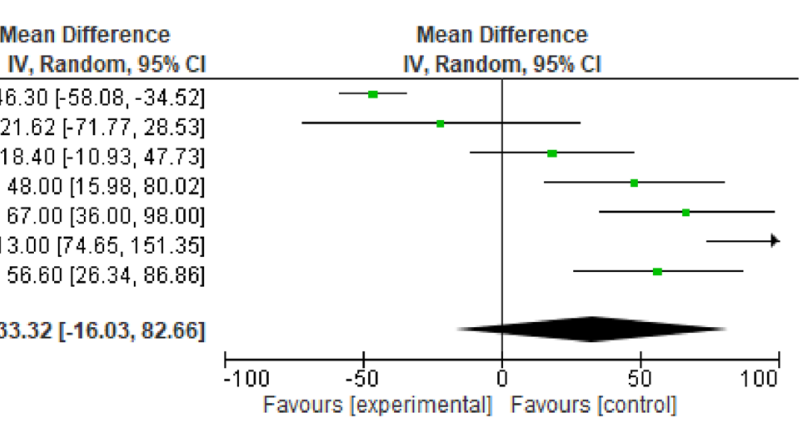

due to its technically complex aspect. Our subgroup analyses showed that RARC plus ICUD in high-volume centers was likely associated with a decreased incidence of postoperative major complications. This finding might exhibit the true impact of ICUD after the learning curve because the matured robotic team, including a skilled mentor and expertise in the patient care, help to shorten or mitigate the effect of the learning curve.

We found that EBL and blood transfusion rates were significantly lower in patients who received an ICUD rather than those who underwent an ECUD in the present study. Recent meta-analyses comparing RARC with ECUD vs. ORC described significantly lower EBL and blood transfusion rates in RARC than in ORC [5, 7, 33, 34], with this tendency being extended in RARC with ICUD in this study. This could be explained by the influence of pneumoperitoneum throughout the surgery and precise dissection due to three-dimensional magnified visualization. Interestingly, even in subgroup analyses of low-volume centers, statistically significant differences regarding EBL and blood transfusion retained between ICUD and ECUD, suggesting that the influence of pneumoperitoneum may outweigh a negative effect on the early phase of the learning curve. Blood transfusion has been shown to be significantly associated with worse perioperative morbidity and survival [38-40]. It was also noteworthy that ICUD patients with high ageadjusted Charlson Comorbidity Index had a lower risk of complications relative to ECUD patients [23]. One possible 
explanation for this could be that ICUD reduced surgical stress, including less blood loss, few transfusion rates, or less incision pain. As such, ICUD may possibly be even more indicated for older or frail patients [41].

Another potential benefit of ICUD is the faster recovery of bowel function due to the avoidance of excessive bowel manipulation and less exposure time to external air. The current meta-analysis showed that ileus was trending in favor of ICUD but failed to prove the statistical significance. The effect of ERAS on outcomes may have corrected for the background noise to assess differences between the UD types. ERAS pathways aim to minimize the physiological stress and facilitate postoperative recovery, which has led to improved perioperative outcomes, including shorter LOS and fewer GI-related complications [42, 43]. A small incision in ICUD may enable less use of opioids, and it is possible that the combination of ICUD and ERAS may provide further improvements in the rate of ileus through synergistic effects [44].

Although ICUD has often been criticized as a time-consuming procedure, we found no difference between ICUD and ECUD in operative time. Note that even when there was no significant difference in operative time by a subgroup analysis of low-volume centers, significant heterogeneity remains to be observed. Thus, in view of unknown cause of heterogeneity possibly occurred from inter-study, interpretation should be cautious. In addition, LN yields were significantly higher in patients treated with ICUD. This suggests a higher surgical proficiency of surgeons preferring ICUD in the published data to date. The determination of the operating technique (intracorporeal or extracorporeal) and UD (ileal conduit or neobladder) is multifactorial. It depends on the patient's and surgeon's preferences as well as the tumor pathologic aspect [45]. Experienced surgeons are likely to choose the most challenging but best possible surgery (i.e., ICUD) which may deliver a more favorable operative time and perioperative outcomes, including blood transfusion rates, EBL, and LN yields.

This systematic review and meta-analysis has some limitations. First, no RCTs were included in this study, which caused considerable selection bias. As mentioned above, the patient's and surgeon's preferences highly affect UD's decision-making. Second, there is some degree of heterogeneity in measuring outcomes. To standardize the reporting methodology, most of the studies reported complications using the Clavien-Dindo system, but few studies reported by the formed reporting system, which was recommended by an ad hoc European Association of Urology panel [46]. Third, despite subgroup analyses stratified by hospital volume, it may not reflect reality. Although IRCC undoubtedly represents a pioneer in the field of robotic surgeries, it consists of 26 centers combining academic and private centers, having been assigned to the low-volume center [25]. Additionally, this study highly affects our several analyses due to a large number of included patients but did not adjust any potential confounding factors inherent in a retrospective design, leading to confound the analyses. Fourth, although the extent of LN dissection is more clinically relevant than LN yields, we could not discuss about the extent of LN dissection due to lack of data in eligible studies. In addition, the cumulative experience of ICUD for each surgeon before the analysis is not unknown. Case selection is another bias that remains uncontrolled for. Finally, since RC is a complex procedure, late complications such as stenosis of uretero-ileal anastomosis are often concerns that remain unassessed. Unfortunately, there are very few available studies on this matter [10].

Beyond the limitations, our meta-analysis has practical implications. Since ICUD is a highly complex procedure, there appears to exist a long learning curve. However, at the cost of the learning curve and prolonged operative time, the data suggest that some perioperative benefits can be obtained for the patients. Shared knowledge, mentorship programs with surgeons and the development of a dedicated team organized around anesthesiologists, intensive care unit staffs and bedside assistants are needed to obtain on the potential benefits of ICUD.

In conclusion, this meta-analysis suggested that complications of RARC with ICUD in the short-term and midterm periods were equivalent to those of ECUD with a trend toward faster bowel recovery. ICUD performed by high-volume centers significantly achieved the reduced risk of postoperative major complications. Furthermore, blood loss and transfusion rates were superior in patients receiving ICUD compared to those receiving ECUD, regardless of hospital volume. Based on these findings, centralization of patients who are candidates for ICUD seems advisable. The best treatment strategies for each individual patient at each institution should be determined, considering patient comorbidities, surgeon's experience and hospital volume. However, since the choice of UD is likely to be subject to a strong bias, interpretation should be cautious. A trial comparing RARC with ICUD and ORC (iROC; clinicaltrials.gov: NCT03049410) in progress is awaited.

Supplementary Information The online version contains supplementary material available at https://doi.org/10.1007/s10147-021-01972-2.

Acknowledgements Ekaterina Laukhtina and Victor M. Schuettfort are supported by the EUSP Scholarship of the European Association of Urology (EAU).

Funding Open access funding provided by Medical University of Vienna. 


\section{Declarations}

Conflict of interest No author has any conflict of interest.

Open Access This article is licensed under a Creative Commons Attribution 4.0 International License, which permits use, sharing, adaptation, distribution and reproduction in any medium or format, as long as you give appropriate credit to the original author(s) and the source, provide a link to the Creative Commons licence, and indicate if changes were made. The images or other third party material in this article are included in the article's Creative Commons licence, unless indicated otherwise in a credit line to the material. If material is not included in the article's Creative Commons licence and your intended use is not permitted by statutory regulation or exceeds the permitted use, you will need to obtain permission directly from the copyright holder. To view a copy of this licence, visit http://creativecommons.org/licenses/by/4.0/.

\section{References}

1. Babjuk M et al (2019) European Association of Urology Guidelines on non-muscle-invasive bladder cancer (TaT1 and carcinoma in situ) —2019 update. Eur Urol 76(5):639-657

2. Svatek RS et al (2011) Discrepancy between clinical and pathological stage: external validation of the impact on prognosis in an international radical cystectomy cohort. BJU Int 107(6):898-904

3. Menon $\mathrm{M}$ et al (2003) Nerve-sparing robot-assisted radical cystoprostatectomy and urinary diversion. BJU Int 92(3):232-236

4. Moschini M et al (2019) Open versus robotic cystectomy: a propensity score matched analysis comparing survival outcomes. J Clin Med 8(8):1192

5. Kimura $\mathrm{S}$ et al (2019) Comparison of perioperative complications and health-related quality of life between robot-assisted and open radical cystectomy: a systematic review and meta-analysis. Int $\mathbf{J}$ Urol 26(8):760-774

6. Iwata T et al (2019) Oncologic outcomes after robot-assisted versus open radical cystectomy: a systematic review and metaanalysis. World J Urol 37(8):1557-1570

7. Soria $\mathrm{F}$ et al (2020) Comparative effectiveness in perioperative outcomes of robotic versus open radical cystectomy: results from a multicenter contemporary retrospective cohort study. Eur Urol Focus 6(6):1233-1239

8. Parekh DJ et al (2018) Robot-assisted radical cystectomy versus open radical cystectomy in patients with bladder cancer (RAZOR): an open-label, randomised, phase 3, non-inferiority trial. Lancet 391(10139):2525-2536

9. Bochner BH et al (2015) Comparing open radical cystectomy and robot-assisted laparoscopic radical cystectomy: a randomized clinical trial. Eur Urol 67(6):1042-1050

10. Presicce F et al (2020) Late complications of robot-assisted radical cystectomy with totally intracorporeal urinary diversion. World J Urol

11. Dason S, Goh AC (2018) Contemporary techniques and outcomes of robotic cystectomy and intracorporeal urinary diversions. Curr Opin Urol 28(2):115-122

12. Sim A et al (2015) Robot-assisted radical cystectomy and intracorporeal urinary diversion-safe and reproducible? Cent Eur J Urol 68(1):18-23

13. Simone $\mathrm{G}$ et al (2018) Perioperative and mid-term oncologic outcomes of robotic assisted radical cystectomy with totally intracorporeal neobladder: results of a propensity score matched comparison with open cohort from a single-centre series. Eur J Surg Oncol 44(9):1432-1438
14. Hussein AA et al (2018) Outcomes of intracorporeal urinary diversion after robot-assisted radical cystectomy: results from the international robotic cystectomy consortium. J Urol 199(5):1302-1311

15. Liberati A et al (2009) The PRISMA statement for reporting systematic reviews and meta-analyses of studies that evaluate healthcare interventions: explanation and elaboration. BMJ 339:b2700

16. Wan X et al (2014) Estimating the sample mean and standard deviation from the sample size, median, range and/or interquartile range. BMC Med Res Methodol 14:135

17. Bruins HM et al (2020) The importance of hospital and surgeon volume as major determinants of morbidity and mortality after radical cystectomy for bladder cancer: a systematic review and recommendations by the European association of urology muscleinvasive and metastatic bladder cancer guideline panel. Eur Urol Oncol 3(2):131-144

18. Hussein AA et al (2020) A comparative propensity score-matched analysis of perioperative outcomes of intracorporeal vs extracorporeal urinary diversion after robot-assisted radical cystectomy: results from the International Robotic Cystectomy Consortium. BJU Int 126(2):265-272

19. Sterne JA et al (2016) ROBINS-I: a tool for assessing risk of bias in non-randomised studies of interventions. BMJ 355:i4919

20. Lenfant $\mathrm{L}$ et al (2018) Perioperative outcomes and complications of intracorporeal vs extracorporeal urinary diversion after robotassisted radical cystectomy for bladder cancer: a real-life, multiinstitutional french study. World J Urol 36(11):1711-1718

21. Bertolo R et al (2019) Perioperative outcomes and complications after robotic radical cystectomy with intracorporeal or extracorporeal ileal conduit urinary diversion: head-to-head comparison from a single-institutional prospective study. Urology 129:98-105

22. Tan TW et al (2019) Safe transition from extracorporeal to intracorporeal urinary diversion following robot-assisted cystectomy: a recipe for reducing operative time, blood loss and complication rates. World J Urol 37(2):367-372

23. Mazzone E et al (2021) Robot-assisted radical cystectomy with intracorporeal urinary diversion decreases postoperative complications only in highly comorbid patients: findings that rely on a standardized methodology recommended by the European Association of Urology Guidelines. World J Urol 39(3):803-812

24. Shim JS et al (2020) Do patients benefit from total intracorporeal robotic radical cystectomy?: a comparative analysis with extracorporeal robotic radical cystectomy from a Korean multicenter study. Investig Clin Urol 61(1):11-18

25. Zhang JH et al (2020) Large single institution comparison of perioperative outcomes and complications of open radical cystectomy, intracorporeal robot-assisted radical cystectomy and robotic extracorporeal approach. J Urol 203(3):512-521

26. Pyun JH et al (2016) Robot-assisted radical cystectomy with total intracorporeal urinary diversion: comparative analysis with extracorporeal urinary diversion. J Laparoendosc Adv Surg Tech A 26(5):349-355

27. Kingo PS et al (2017) Postoperative C-reactive protein concentration and clinical outcome: comparison of open cystectomy to robot-assisted laparoscopic cystectomy with extracorporeal or intracorporeal urinary diversion in a prospective study. Scand J Urol 51(5):381-387

28. Pruthi RS et al (2010) Robotic-assisted laparoscopic intracorporeal urinary diversion. Eur Urol 57(6):1013-1021

29. Aboumohamed AA et al (2014) Health-related quality of life outcomes after robot-assisted and open radical cystectomy using a validated bladder-specific instrument: a multi-institutional study. Urology 83(6): 1300-1308

30. Mistretta FA et al (2021) Robot-assisted radical cystectomy for nonmetastatic urothelial carcinoma of urinary bladder: a comparison between intracorporeal versus extracorporeal orthotopic ileal neobladder. J Endourol 35(2):151-158 
31. Hussein AA et al (2019) Ten-year oncologic outcomes following robot-assisted radical cystectomy: results from the international robotic cystectomy consortium. J Urol 202(5):927-935

32. Venkatramani V et al (2020) Predictors of recurrence, and progression-free and overall survival following open versus robotic radical cystectomy: analysis from the RAZOR trial with a 3-year followup. J Urol 203(3):522-529

33. Satkunasivam R et al (2019) Robot-assisted radical cystectomy versus open radical cystectomy: a meta-analysis of oncologic, perioperative, and complication-related outcomes. Eur Urol Oncol 2(4):443-447

34. Tzelves L et al (2019) Does the use of a robot decrease the complication rate adherent to radical cystectomy? A systematic review and meta-analysis of studies comparing open with robotic counterparts. J Endourol 33(12):971-984

35. Hayn MH et al (2010) The learning curve of robot-assisted radical cystectomy: results from the International Robotic Cystectomy Consortium. Eur Urol 58(2):197-202

36. Porreca A et al (2020) Robot-assisted radical cystectomy with totally intracorporeal urinary diversion: surgical and early functional outcomes through the learning curve in a single high-volume center. J Robot Surg 14(2):261-269

37. Moschini $\mathrm{M}$ et al (2016) Critical review of outcomes from radical cystectomy: can complications from radical cystectomy be reduced by surgical volume and robotic surgery? Eur Urol Focus 2(1):19-29

38. Moschini M et al (2015) Effect of allogeneic intraoperative blood transfusion on survival in patients treated with radical cystectomy for nonmetastatic bladder cancer: results from a single high-volume institution. Clin Genitourin Cancer 13(6):562-567
39. Sari Motlagh R et al (2020) Impact of hospital and surgeon volumes on short-term and long-term outcomes of radical cystectomy. Curr Opin Urol 30(5):701-710

40. Kluth LA et al (2014) Impact of peri-operative blood transfusion on the outcomes of patients undergoing radical cystectomy for urothelial carcinoma of the bladder. BJU Int 113(3):393-398

41. Shariat SF, Milowsky M, Droller MJ (2009) Bladder cancer in the elderly. Urol Oncol 27(6):653-667

42. Pozo C et al (2019) Enhanced recovery after radical cystectomy. Curr Opin Urol 29(3):227-238

43. Miller $C$ et al (2017) Introduction of robot-assisted radical cystectomy within an established enhanced recovery programme. BJU Int 120(2):265-272

44. Tan WS et al (2018) Intracorporeal robot-assisted radical cystectomy, together with an enhanced recovery programme, improves postoperative outcomes by aggregating marginal gains. BJU Int 121(4):632-639

45. Lee RK et al (2014) Urinary diversion after radical cystectomy for bladder cancer: options, patient selection, and outcomes. BJU Int 113(1):11-23

46. Mitropoulos D et al (2012) Reporting and grading of complications after urologic surgical procedures: an ad hoc EAU guidelines panel assessment and recommendations. Eur Urol 61(2):341-349

Publisher's Note Springer Nature remains neutral with regard to jurisdictional claims in published maps and institutional affiliations.

\section{Authors and Affiliations}

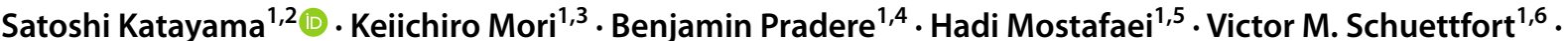 Fahad Quhal $^{1,7}$. Reza Sari Motlagh ${ }^{1,8}$. Ekaterina Laukhtina ${ }^{1,9} \cdot$ Marco Moschini $^{10}$. Nico C. Grossmann ${ }^{1,11}$. Yasutomo Nasu ${ }^{2} \cdot$ Shahrokh F. Shariat ${ }^{1,9,12,13,14,15,16} \cdot$ Harun Fajkovic ${ }^{1,15}$}

1 Department of Urology, Comprehensive Cancer Center, Vienna General Hospital, Medical University of Vienna, Währinger Gürtel 18-20, 1090 Vienna, Austria

2 Department of Urology, Okayama University Graduate School of Medicine, Dentistry and Pharmaceutical Sciences, Okayama, Japan

3 Department of Urology, The Jikei University School of Medicine, Tokyo, Japan

4 Department of Urology, University Hospital of Tours, Tours, France

5 Research Center for Evidence Based Medicine, Tabriz University of Medical Sciences, Tabriz, Iran

6 Department of Urology, University Medical Center Hamburg-Eppendorf, Hamburg, Germany

7 Department of Urology, King Fahad Specialist Hospital, Dammam, Saudi Arabia

8 Men's Health and Reproductive Health Research Center, Shahid Beheshti University of Medical Sciences, Tehran, Iran
9 Institute for Urology and Reproductive Health, Sechenov University, Moscow, Russia

10 Department of Urology, Luzerner Kantonsspital, Luzern, Switzerland

11 Department of Urology, University Hospital Zurich, Zurich, Switzerland

12 Department of Urology, Weill Cornell Medical College, New York, NY, USA

13 Department of Urology, University of Texas Southwestern, Dallas, TX, USA

14 Department of Urology, Second Faculty of Medicine, Charles University, Prague, Czech Republic

15 Karl Landsteiner Institute of Urology and Andrology, Vienna, Austria

16 Division of Urology, Department of Special Surgery, Jordan University Hospital, The University of Jordan, Amman, Jordan 